\title{
Magnetoimpedance Effect in CoFeMoSiB As-Quenched and Surface Modified Amorphous Ribbons in the Presence of Igon Oxide Nanoparticles of Water-Based Ferrofluid
}

\author{
Zahra Lotfollahi, ${ }^{1}$ Ahmad Amirabadizadeh, ${ }^{1}$ Aleksander P. Safronov, ${ }^{2,3}$ \\ Igor V. Beketov, ${ }^{2,3}$ and Galina V. Kurlyandskaya ${ }^{2,4}$ \\ ${ }^{1}$ University of Birjand, Birjand 97175-615, Iran \\ ${ }^{2}$ Ural Federal University, Ekaterinburg 620083, Russia \\ ${ }^{3}$ Institute of Electrophysics UD RAS, Ekaterinburg 620016, Russia \\ ${ }^{4}$ University of the Basque Country (UPV/EHU), 48940 Leioa, Spain \\ Correspondence should be addressed to Galina V. Kurlyandskaya; galina@we.lc.ehu.es
}

Received 19 June 2017; Accepted 12 September 2017; Published 16 October 2017

Academic Editor: Andrea Cusano

Copyright (c) 2017 Zahra Lotfollahi et al. This is an open access article distributed under the Creative Commons Attribution License, which permits unrestricted use, distribution, and reproduction in any medium, provided the original work is properly cited.

\begin{abstract}
Giant magnetoimpedance (GMI) has been proposed as a powerful technique for biosensing. In GMI biosensors based on the magnetic label detection the change of the impedance of sensitive element under the application of an external magnetic field was analyzed in the presence of magnetic nanoparticles in a test solution. Amorphous ribbon-based GMI biodetectors have an advantage of low operation frequency and low cost. In this work, magnetic and GMI properties of amorphous $\mathrm{Co}_{68.6} \mathrm{Fe}_{3.9} \mathrm{Mo}_{3.0} \mathrm{Si}_{12.0} \mathrm{~B}_{12.5}$ ribbons were studied in as-quenched and surface modified states both without and in the presence of maghemite ferrofluid. After the surface modification the coercivity was slightly increased and saturation magnetization decreased in good agreement with increase of the surface roughness, a decrease of magnetic elements concentrations in the surface layer, and formation of a surface protective oxide layer. The GMI difference for as-quenched ribbons in absence and in the presence of ferrofluid was measurable for the frequency range of 2 to $10 \mathrm{MHz}$ and the current intensities of 1 to $20 \mathrm{~mA}$. Although the proposed surface modification by the ultrasound treatment did not improve the sensitivity limit for ferrofluid detection, it did not decrease it either.
\end{abstract}

\section{Introduction}

Giant magnetoimpedance is a very high change of the total impedance of ferromagnetic conductor under application of an external magnetic field [1]. GMI effect in soft magnetic materials seems to be quit promising for applications in the technological area of small magnetic field sensors and magnetic biosensors $[2,3]$. The idea of a biosensor based on amorphous ribbon is attractive since this would allow construction of a cheap detector with the sensitive element designed as a disposable strip. GMI has been proposed as a powerful technique for biosensing based on the magnetic label detection or label-free sensing process. In the first case the change of the impedance of sensitive element under the application of an external magnetic field is analyzed in the presence of magnetic nanoparticles (MNPs) in a test solution and the origin of the sensitivity is clearly connected with the stray fields crated by the MNPs [3,4]. In the second case if rapidly quenched ribbon sensitive element is employed the advantage of natural nonuniform magnetic anisotropy was taken [5]. One of the possible mechanisms for label-free biosensing could be the difference of the corrosive properties of biofluids and their changes in course of living system life cycle.

Such a parameter as surface roughness is very important in the case of amorphous ribbon-based biosensors of both types. On one hand, it might be seen as an obstacle in the case of magnetic label detection as an obstacle influencing the response in the strong skin effect condition. On the other hand, one can expect the improvement of the magnetic flux closing for the rough surface with magnetic ferrofluid spread on it. It was shown recently that naturally formed 
or artificially designed surface defects can be used for the control of the GMI sensitivity [6]. Mechanical processing of the surface modification of the amorphous materials is practically impossible due to their physical properties. For the magnetic detection of the MNPs they usually select Mo or Cr doped compositions with enhanced corrosion stability. Consequently, chemical processing of these kinds of ribbons (like lithographic process including chemical etching) is also not simple. Searching for the methods of creation of well defined artificially designed surface defects for the ribbons with enhanced corrosion stability is therefore a challenge.

In an amorphous ribbon, the GMI phenomenon was explained long ago as a consequence of the skin effect: the increase of the effective magnetic permeability $\left(\mu_{\mathrm{ef}}\right)$ leads to a decrease of the classic skin penetration depth $(\delta)$ and an increase of the impedance. According to the expression for the penetration depth one can write [1]

$$
\delta=\frac{1}{\left(f \sigma \mu_{\mathrm{ef}}\right)^{1 / 2}},
$$

where $\sigma$ is the electrical conductivity and $\mu$ is transverse dynamic magnetic permeability. Previous studies showed that the GMI responses in appropriate conditions can be clearly dependent on the presence of the magnetic ferrofluid and the parameters of the driving current $[3,6]$. The shape or particular geometry of the sensitive element strongly influences the GMI behavior. The surface roughness and irregularities in the surface composition or stresses and associated with them variations in local magnetic anisotropy can play an important role contributing to the effective magnetic anisotropy peculiarities and GMI behavior $[6,7]$.

Another attractive research branch of surface modification of amorphous ribbons has been developed recently counting ion irradiation, chemical polishing, and diamagnetic or magnetic material coating [8]. It was shown that chemical thinning or polishing can significantly change the GMI responses of the amorphous ribbon-based sensitive elements. The superior GMI values were observed for polished samples and were interpreted as magnetic field induced changes on the bases of skin depth model [9]. The surface roughness of the sample is important when the skin effect is strong. Microstructure and magnetic and MI properties of $\mathrm{Co}_{73} \mathrm{Fe}_{4.5} \mathrm{Mn}_{0.5} \mathrm{Nb}_{1.0} \mathrm{Si}_{4.2} \mathrm{~B}_{16.8}$ ribbon with and without artificial defects were studied in [6]. In this work the GMI value has been decreased with increasing the density of holes and the anisotropy induced by artificial defects strongly affected GMI properties. Enhancement of GMI in polished samples is attributed to smoother surface of the ribbons which reduces the effective anisotropy [6]. Treating the surface of a ribbon with an appropriate concentration of acid has been shown to be a useful way to improve the sensitivity of detection of a ribbon-based GMI biosensor, which is probably related to effect of the microholes-pattern produced by the acid on the surface of ribbon $[5,10]$.

In this work, the GMI responses of as-quenched $\mathrm{Co}_{68.6} \mathrm{Fe}_{3.9} \mathrm{Mo}_{3.0} \mathrm{Si}_{12.0} \mathrm{~B}_{12.5}$ ribbon in initial state and after surface modification by acid in ultrasonic bath were studied in the presence of water-based ferrofluid (FF) at different alternating current $(\mathrm{AC})$ frequencies and intensities.

\section{Materials and Methods}

Amorphous ribbons with nominal composition of $\mathrm{Co}_{68.6} \mathrm{Fe}_{3.9} \mathrm{Mo}_{3.0} \mathrm{Si}_{12.0} \mathrm{~B}_{12.5}$ with $0.7 \mathrm{~mm}$ width and $20 \mu \mathrm{m}$ thickness and length of few meters were prepared by rapid quenching technique [11, 12]. This material was selected on the basis of previously studied physical properties and especially combination of high GMI and enhanced corrosion stability. Saturation magnetostriction of this ribbon was positive and quite small $\left(\lambda_{s} \cong+0.01 \times 10^{-7}\right)$ [12]. First of all pure elements $(\mathrm{Fe}, \mathrm{Co}, \mathrm{Mo}, \mathrm{B}$, and $\mathrm{Si}$ ) underwent induction alloying in an Ar atmosphere. From the master alloy ingots, amorphous ribbon samples of about $25 \mathrm{~mm}$ thickness and $0.7 \mathrm{~mm}$ width were produced by melt spinning onto $\mathrm{Cu}$ wheel at tangential velocity of $\sim 30 \mathrm{~m} / \mathrm{s}$. The molten metal was ejected by argon at an overpressure of $250 \mathrm{mbar}$ through the orifice in the quartz nozzle placed at a distance of about $0.2 \mathrm{~mm}$ from the wheel surface.

Iron oxide magnetic nanoparticles (MNPs) were synthesized by the laser target evaporation (LTE) - the highly productive method of physical dispersion based on the evaporation of a solid pellet by laser beam with consequent condensation of vapors in the gas phase [13, 14]. Special advantages of this technique are the big size of the single batch and spherical shape of the MNPs $[15,16]$. The phase structure of MNPs and amorphous state of the ribbons were characterized by X-ray diffraction (XRD) using PANalytical X'Pert PRO X-ray Diffractometer with $\mathrm{Cu}-\mathrm{K}_{\alpha}$ radiation (wave length $\lambda=1.5418 \AA$ ). The Bruker software TOPAS-3 with Rietveld full-profile refinement was employed for the quantitative analysis (the unit cell parameters, peak shape, background, systematic $2 \theta$ shift, and displacement). In addition, the average size of the coherent diffraction domains was estimated by the Scherrer approach. Transmission electron microscopy (TEM) was performed by JEM 2100 microscope at $200 \mathrm{kV}$ accelerating voltage.

Electrostatically stabilized ferrofluids were prepared by ultrasound treatment using sodium citrate solutions ( $5 \mathrm{mM}$ ) in distilled water. Stock dispersion was $6 \%$ considering MNPs and $0.2 \%$ considering citrate. Dispersion was exhaustively treated by ultrasound: $250 \mathrm{~min}$ in a laboratory ultrasound bath and then $30 \mathrm{~min}$ in Cole-Palmer ultrasound processor CPX-750 operated at $250 \mathrm{~W}$ output. Then the dispersion was centrifuged at $8000 \mathrm{rpm}$ for $5 \mathrm{~min}$. The supernatant was taken out and used further. The combined concentration of the supernatant ferrofluid was 5.2\% including MNPs and citrate. Presuming that the concentration of citrate does not change in the treatment the final concentration of MNPs in ferrofluid was $5.0 \%$.

Surface modification of the amorphous ribbon was done in an ultrasonic bath with $5 \% \mathrm{H}_{3} \mathrm{PO}_{3}$ acid concentration during 120 minutes' treatment for creation of microholes as artificial surface defects. Surface features before and after modifications were studied by the scanning electron microscopy (SEM) using by TM3000 HITACHI in the secondary electron mode. Magnetic measurements to study the magnetic properties of the ribbons and MNPs at room temperature were done by vibrating sample magnetometer (VSM, Lake Shore 7404). The impedance $Z$ is measured by the four-point 


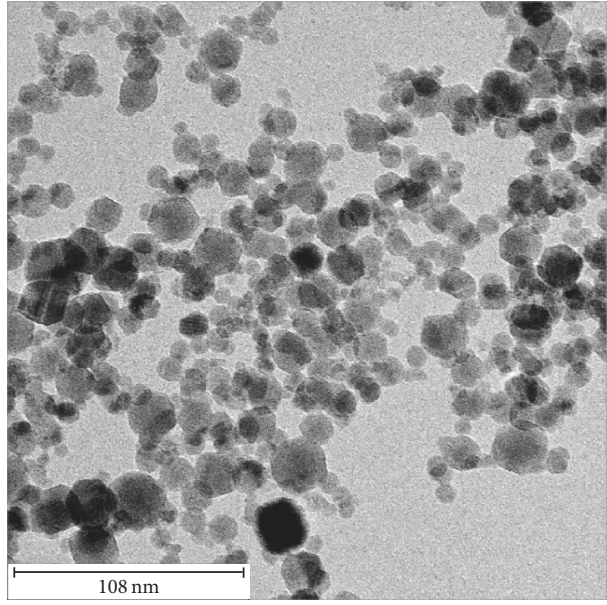

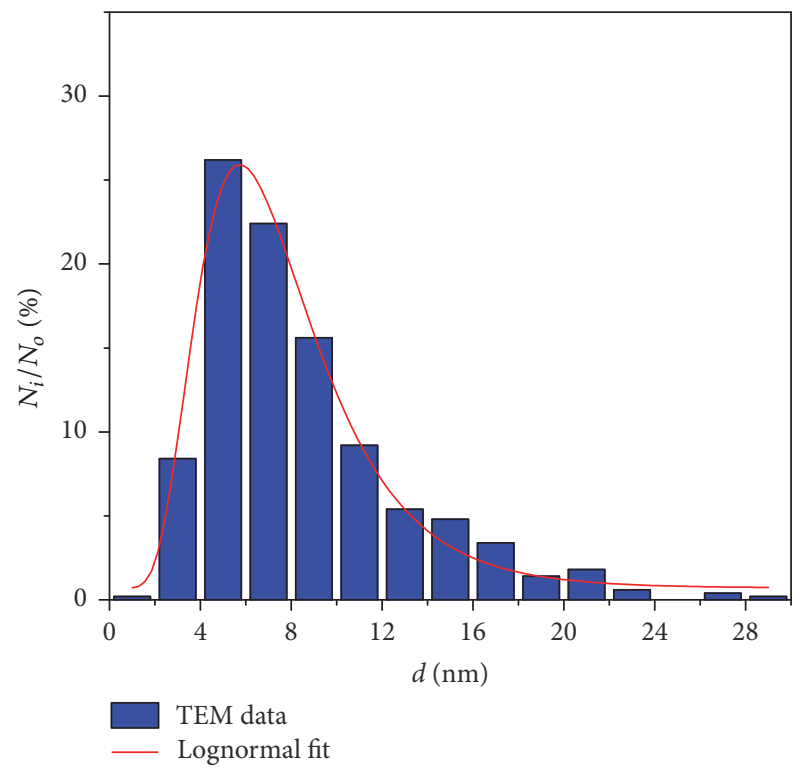

(b)

FIGURE 1: (a) TEM image of maghemite magnetic nanoparticles dried from ferrofluid and (b) particle size distribution of maghemite magnetic nanoparticles obtained on the TEM basis.

method and different frequency $(0.5-10 \mathrm{MHz})$ of the driving AC current $\left(I_{\mathrm{pp}}=1-20 \mathrm{~mA}\right.$, where $I_{\mathrm{pp}}$ is a peak-to-peak intensity) amplitudes was tested in the maximum external magnetic field $H_{\max }= \pm 200$ Oe. Giant magnetoimpedance ratio for total impedance was defined as follows: $\Delta Z / Z=$ $100 \times\left(Z(H)-Z\left(H_{\max }\right)\right) / Z\left(H_{\max }\right)$. For GMI measurements in presence of water-based ferrofluid with MNPs of the iron oxide we selected $3.5 \mathrm{~cm}$ long $\mathrm{Co}_{68.6} \mathrm{Fe}_{3.9} \mathrm{Mo}_{3.0} \mathrm{Si}_{12.0} \mathrm{~B}_{12.5}$ amorphous ribbon with the thickness of 20 microns and widths of about $0.7 \mathrm{~mm}$. Amorphous ribbon was placed into a plastic tube with $30 \mathrm{~mm}$ length and $1 \mathrm{~mm}$ diameter. It was located in center of a tube filled with ferrofluid. More details on GMI experimental setup can be found in [17].

\section{Results and Discussions}

Figure 1(a) shows TEM image of air-dry iron oxide MNPs obtained by LTE technique. The MNPs were nonagglomerated and their shape was close to be spherical. Very few of the particles appeared to have hexagonal corners. Particle size distribution (Figure 1(b)) calculated using the TEM data was lognormal with the median value $d_{0}=11.7 \mathrm{~nm}$ and the dispersion $\sigma=0.423$.

The XRD pattern (Figure 2(a)) of the air-dried MNPs shows the crystallite structure of MNPs corresponded to the inverse spinel lattice with a space group $\mathrm{Fd} 3 \mathrm{~m}$. The airdry MNPs contained only one crystalline phase of ferrite with a mean crystallite size of $20 \mathrm{~nm}$. The slightly smaller size of magnetic nanoparticles dried from ferrofluid is not surprising as the coarse fraction of the nanoparticles was partly removed during ferrofluid preparation. The lattice parameter was found to be $a=0.8358 \mathrm{~nm}$, which was larger than that for maghemite $\left(\gamma-\mathrm{Fe}_{2} \mathrm{O}_{3}, a=0.8346 \mathrm{~nm}\right)$ but lower than that for magnetite $\left(\mathrm{Fe}_{3} \mathrm{O}_{4}, a=0.8396\right)$ [18]. Based on the dependence between the lattice period of the spinel cell and the effective state of oxidation of $\mathrm{Fe}$ the composition of MNPs contained 76\% of $\gamma-\mathrm{Fe}_{2} \mathrm{O}_{3}$ and $24 \%$ of $\mathrm{Fe}_{3} \mathrm{O}_{4}$. The coherence length of monocrystalline domains estimated using the Scherrer approach was $11 \mathrm{~nm}$. This value correlates well with the median value of PSD $(11.7 \mathrm{~nm})$ obtained by TEM.

The XRD pattern of the as-quenched ribbon (Figure 2(b)) confirmed the absence of the long range ordering and crystalline phases: a clear amorphous structure and broad diffraction peak were observed.

The SEM micrographs (Figures 3(a) and 3(b)) of asquenched and surface modified amorphous ribbons show the surface morphology before and after ultrasound treatment in orthophosphoric acid. SEM studies revealed sufficiently smooth surface of the bright side of as-quenched amorphous ribbon with typical for rapid quenching technique thickness variation of this kind of samples. The longitudinal lines in the direction of the ribbon long side are defects of the thickness variation formed during the solidification [19]. Figure 3(b) shows the morphology of surface defects which roughly can be described as round indentations with average diameter of about 150 micrometers. No anisotropy in the shape of the defects or their orientation was observed but surface defects were equidistantly separated from each other as a result of self-assembling process. Very rough estimation shows that approximately $50 \%$ of the surface of the ribbon is covered by the surface defects. It is also clear that the surface layer of about $2-3 \mu \mathrm{m}$ is most affected part of the ribbon in the course of the ultrasound treatment: the scheme of the changes is shown on Figure 3(c). 


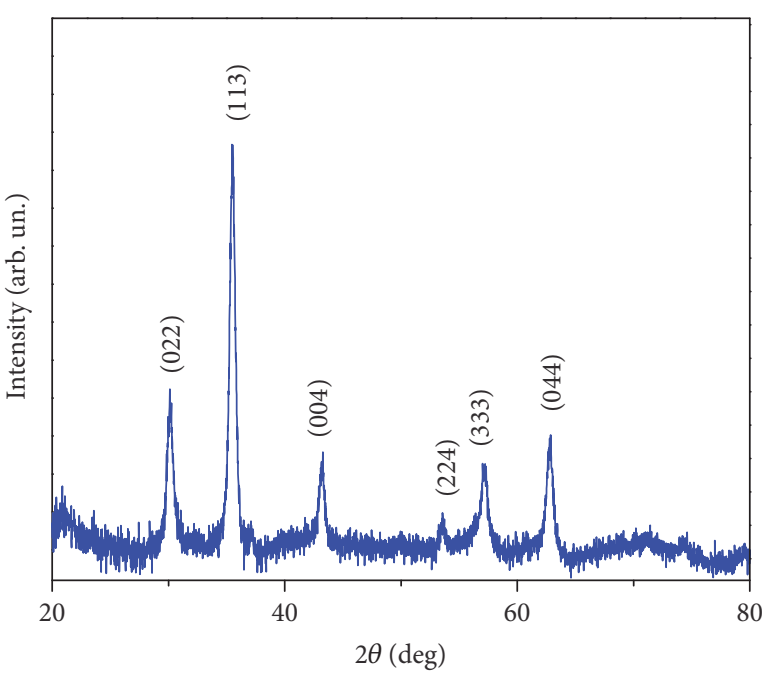

(a)

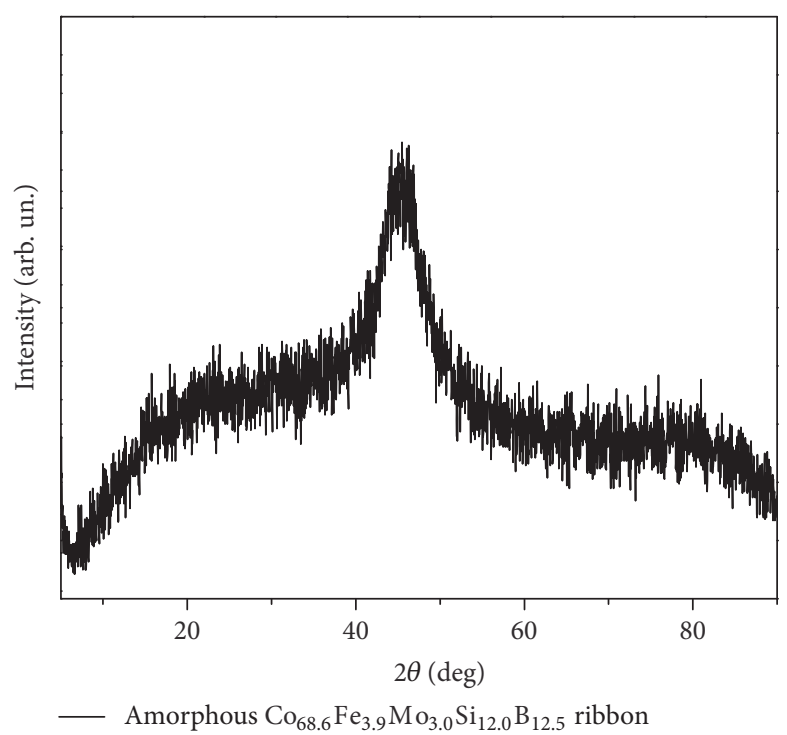

(b)

FIGURE 2: XRD pattern of (a) air-dried magnetic nanoparticles and (b) amorphous $\mathrm{Co}_{68.6} \mathrm{Fe}_{3.9} \mathrm{Mo}_{3.0} \mathrm{Si}_{12.0} \mathrm{~B}_{12.5}$ ribbon.

The changes of the structure of the surface modified amorphous ribbon can be summarized as follows. Ultrasound treatment results in appearance of round indentations with average diameter of about 150 micrometers and few $\mu \mathrm{m}$ in depth. It is known that surface layer of rapidly quenched ribbons may have different composition, stress distribution, and effective magnetic anisotropy peculiarities $[5,20]$. We propose simple understanding of the surface modification. Surface layer is passivated via oxides layer formation which starts near surface defects created during solidification. At certain point thick oxide patch (having different mechanical properties in comparison with metallic glass part) breaks creating indentation defect.

Figure 4 shows the hysteresis loops of the amorphous ribbons before and after surface modification. Magnetic hysteresis loops of ferrofluid and MNPs are shown in Figure 5. Magnetic characterization results for as-quenched (AQ) and surface modified (SM) ribbons, MNPs, and ferrofluid are summarized in Table 1. Data collected in Table 1 and Figure 4 show that as-quenched amorphous ribbons are soft ferromagnetic materials with very low coercivity, low saturation field, and longitudinal effective magnetic anisotropy. The obtained value of saturation magnetization is similar to that one obtained for ribbons of close compositions [21].

Surface modification by the controlled ultrasound treatment in acid results in a slight decrease of saturation magnetization from 87 to $76 \mathrm{emu} / \mathrm{g}$ and does not affect the coercivity very much. Slight $M_{s}$ decrease is consistent with surface modification which includes the removal of the part of the initial surface layer and formation of a passivation oxide layer with much lower saturation magnetization comparing with the material of the as-quenched ribbon. The observed behavior seems to be in a good agreement with the increase of the surface roughness, a decrease of the magnetic elements
TABLE 1: Magnetic characterization of the samples. $H_{c}$ : coercivity and $M_{s}$ : saturation magnetization.

\begin{tabular}{lcc}
\hline Sample & $H_{c}(\mathrm{Oe})$ & $M_{s}(\mathrm{emu} / \mathrm{g})$ \\
\hline As-quenched ribbon $(\mathrm{AQ})$ & $0.3 \pm 0.1$ & $87 \pm 3$ \\
Surface modified ribbon $(\mathrm{SM})$ & $0.4 \pm 0.1$ & $76 \pm 3$ \\
Magnetic nanoparticles & $26.7 \pm 0.5$ & $57 \pm 2$ \\
Ferrofluid & $\sim 0.5$ & $2.0 \pm 0.1$ \\
\hline
\end{tabular}

concentration in the surface layer, and formation of a surface protective oxide layer.

Also we can see that $M_{s}$ of the MNPs is about $57 \mathrm{emu} / \mathrm{g}$. This value is lower than that one of the bulk magnetite or maghemite as it was previously observed for the MNPs of the observed average size due to nanoscaling effects [16, 17]. At the same time magnetic measurements confirm well the concentration of the MNPs of ferrofluid previously defined by chemical titration technique $[16,22]$. Lower coercivity of the ferrofluid was observed. It is due to the fact that the coarse fraction of the nanoparticles can be partly removed during ferrofluid preparation. The observed coercivity of the order of 0.5 Oe for ferrofluid is consistent with usually obtained values for diluted well separated superparamagnetic particles [16].

It is widely discussed that for GMI biodetection based on the evaluation of the stray fields created by magnetic labels it is important to estimate the maximum value of the stray fields in the work field interval [23]. At the same time rough surface magnetization can be changed by the better closing of the magnetic flux based on the formation of MNPs agglomerates in the shape of chains under application of an external magnetic field [24]. This can be viewed as different mechanism explaining GMI sensitivity to the presence of 


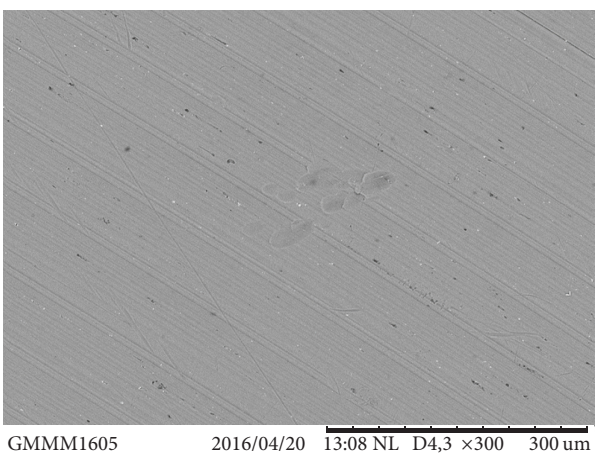

(a)

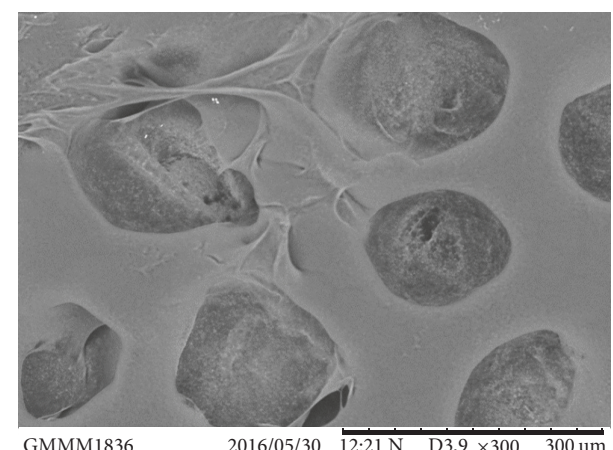

(b)
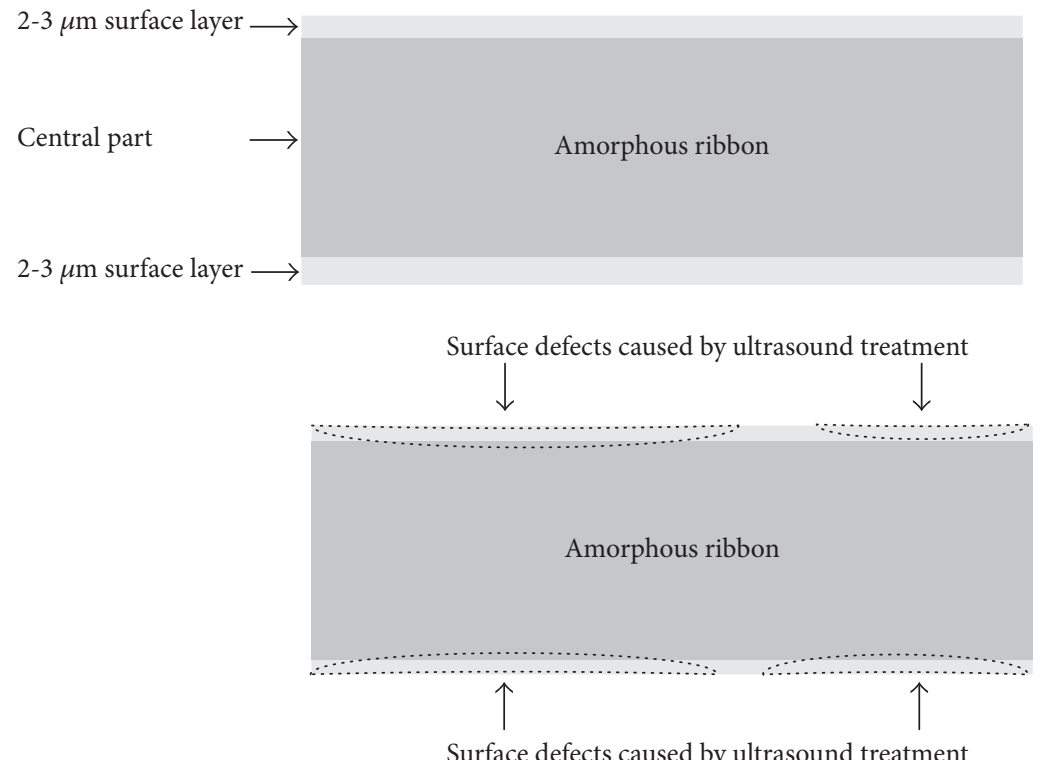

(c)

FIGURE 3: SEM images of the typical surface morphology of the bright side of the $\mathrm{Co}_{68.6} \mathrm{Fe}_{3.9} \mathrm{Mo}_{3.0} \mathrm{Si}_{12.0} \mathrm{~B}_{12.5}$ amorphous ribbons (a) before and (b) after ultrasound surface modification and (c) the scheme of the cross-section features of the surface modified ribbon.

MNPs of ferrofluid in comparison with the stray fields of MNPs contribution.

Figures 6(a) and 6(b) show frequency dependence of the maximum GMI ratio $\left((\Delta Z / Z)_{\max }\right)$ for as-quenched and surface modified ribbons in the presence and absence of ferrofluid for different intensities of the driving current. The results at different alternating current densities show that until $f=5 \mathrm{MHz},(\Delta Z / Z)_{\max }$ increases and after this frequency it changes very little for all intensities of the driving current under consideration. This behavior is consistent with calculated skin depth (see (1)): the condition of strong skin effect for the ribbon of this geometry appears for the frequencies above $4 \mathrm{MHz}$ when the skin depth value becomes less than the half of the ribbon thickness.

In a whole frequency range $0.5-10 \mathrm{MHz}$ GMI-frequency responses of the as-quenched and surface modified ribbons had very similar shapes but the values of the maximum GMI ratios were slightly higher for as-quenched ribbons. Keeping in mind the similarity of the $M(H)$ hysteresis loops one can assign such a difference to the difference in the surface magnetic anisotropy and dynamic magnetic permeability which can be different from the static one. An increase of the surface roughness due to formation of the round indentations with average diameter of about 150 micrometers can be a reason of slight decrease of $(\Delta Z / Z)_{\max }$ value as well as the partial removal of very thin surface layer during ultrasound treatment. The general rule of the lower the local effective magnetic anisotropy distribution the higher the GMI [7] predicts the observed behavior.

$(\Delta Z / Z)_{\max }(f)$ curves appeared to be very much affected by the presence of ferrofluid in AQ and much less in SM ribbon cases. For AQ ribbons in the presence of ferrofluid these curves become lower in comparison with $(\Delta Z / Z)_{\max }(f)$ curves in absence of the ferrofluid (Figure 6(a)). Covering by the ferrofluid resulted in a decrease of GMI ratio in comparison with response in absence of MNPs in the entire frequency range. As usual, the change in the GMI ratio due to the presence of the MNPs can be explained by the effect of their fringe fields on the superposition of the applied axial direct current magnetic field and the induced 


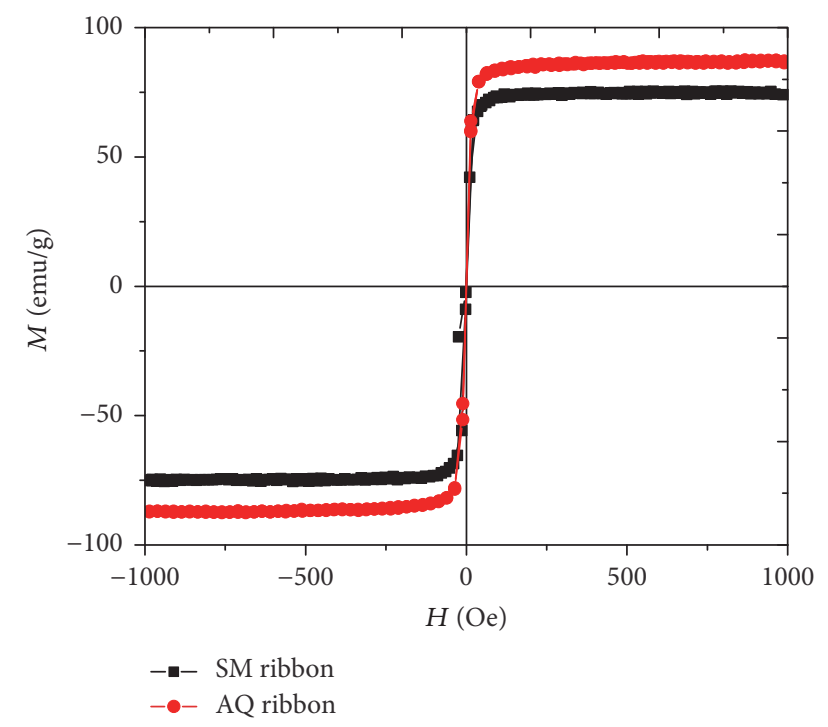

FIGURE 4: Magnetic hysteresis loops of as-quenched (AQ) and surface modified (SM) $\mathrm{Co}_{68.6} \mathrm{Fe}_{3.9} \mathrm{Mo}_{3.0} \mathrm{Si}_{12.0} \mathrm{~B}_{12.5}$ amorphous ribbons.

transverse alternating current field created by the driving current flowing along the axis of the ribbon.

In order to gain better understanding let us analyze the field dependence of GMI ratio. The general shapes of the $\Delta Z / Z(H)$ curves are shown in Figure 7. In both cases (AQ and SM ribbons) the shape of the $\Delta Z / Z(H)$ curves corresponds well to the longitudinal effective magnetic anisotropy (one-peak GMI curve) [7]. At the same time the single peak GMI curves have peculiarity in a very low field: the appearance of very small double peak related to small contribution of the surface component of transverse magnetic anisotropy. Increasing the frequency from $3 \mathrm{MHz}$ to $9 \mathrm{MHz}$ results in the change of the shape of $\Delta Z / Z(H)$ curves and double peak behaviors become more obvious and the dip of the double peak at zero field increases with the frequency increase. Such a change with the frequency increase is consistent with the existence of the contribution of the transverse anisotropy component in rapidly quenched amorphous ribbons without additional heat treatments: the increase of the frequency causes the skin depth decrease and increase of the surface related volume fraction of the effective volume affected by the flowing current.

Let us now analyze the role of the ferrofluid. For the frequency of $f=3 \mathrm{MHz}$ maximum GMI in presence of FF decreases from $65 \%$ to $46 \%$ and for surface modified ribbon decreases from $53 \%$ to $43 \%$. This means that GMI response of $\mathrm{AQ}$ ribbon offers more stable detection opportunities with better noise-to-signal characteristics. Also for frequency $f=$ $9 \mathrm{MHz}$ similar conclusion can be made as the maximum GMI in presence of ferrofluid for ribbon decreases from $85 \%$ to $61 \%$ and for surface modified ribbon decreases from $78 \%$ to $68 \%$. Therefore surface modification and presence of ferrofluid have effect on decreasing of GMI.

If we define sensitivity of sensor as the change in the maximum GMI ratio due to the presence of MNPs with $\Delta \eta=$ $\left(\mathrm{GMI}_{\max }\right)_{(\mathrm{ribbon})}-\left(\mathrm{GMI}_{\max }\right)_{(\mathrm{ribbon}+\mathrm{FF})}[25]$ this parameter at frequencies $f=3 \mathrm{MHz}$ and $f=9 \mathrm{MHz}$ for as-quenched ribbon is about $22 \%$ and for surface modified ribbon is about $10 \%$. This shows that as-quenched ribbon is more sensitive than surface modified ribbon for the modification procedure under consideration.

On-purpose creation of round indentations with average diameter of about 150 micrometers makes ribbon less sensitive to the presence of ferrofluid but the origin of this sensitivity is not trivial. The GMI sensing process connected with three magnetic fields: the AC magnetic field of the alternating current passing through the ribbon, the constant external magnetic field applied during GMI measurements for the change of magnetic permeability of the sensitive element, and the stray fields created by MNPs and round indentations. Surface modification changes feature of the demagnetizing field as small naturally formed defect corresponding to rapid solidification are removed but new defects in the shape of indentations are formed. As the indentations are quite large, comparing with typical quenching defects but out of the border of the round defect the surface roughness becomes even smaller and we can suppose that effective flux closing by the magnetic chains of FF is more effective for the as-quenched ribbon. As the next step one can plan to create smaller equidistant defects using different parameters of ultrasound treatments.

Let us now have a look on the obtained results from the point of view of possible biomedical applications. Although magnetic label detection in the regime of immobilized markers was shown to be most accurate protocol, the possibility of free label detection was also widely discussed $[4,25]$. Successful detection of the magnetic labels is possible only when a number of requests are satisfied. One of the very important requests is the geometrical/dimensional compatibility of the labels and GMI element or element's surface structural features. Biocomponents of the interest in a magnetic biosensing include small molecules, macromolecules, magnetite nanoparticles synthesized by bacteria, viruses and small bacteria, large bacteria, and small and large cells. In the large three cases GMI sensitive elements with on-purpose created surface geometry are desirable. A comparison of the characteristic sizes of some of these biocomponents with $150 \mu \mathrm{m}$ size of self-assembled round indentations (Figure 8) demonstrates that each defect may accommodate certain number of large biocomponents depending on their size: of the order of 270 for Exophiala nigrum (black yeast) [26], about 90 for mesenchymal stem cells [27, 28], and 10-12 for human embryonic kidney cells [23]. If these biocomponents were grown in the presence of MNPs [23] they can be used for evaluation of the iron oxide concentration related to the internalized MNPs (mesenchymal stem cells or human embryonic kidney cells) or MNPs made to adhere to the cell membrane (black yeasts).

In this case due to interaction between magnetic nanoparticles agglomerated in the biological samples in accordance with their natural structure the magnetic flux can be closed in a different way comparing with ferrofluid testing. The result of the flux closing should be dependent on the MNPs concentration in each biological element and the number of elements accommodated in artificial round surface defect. 


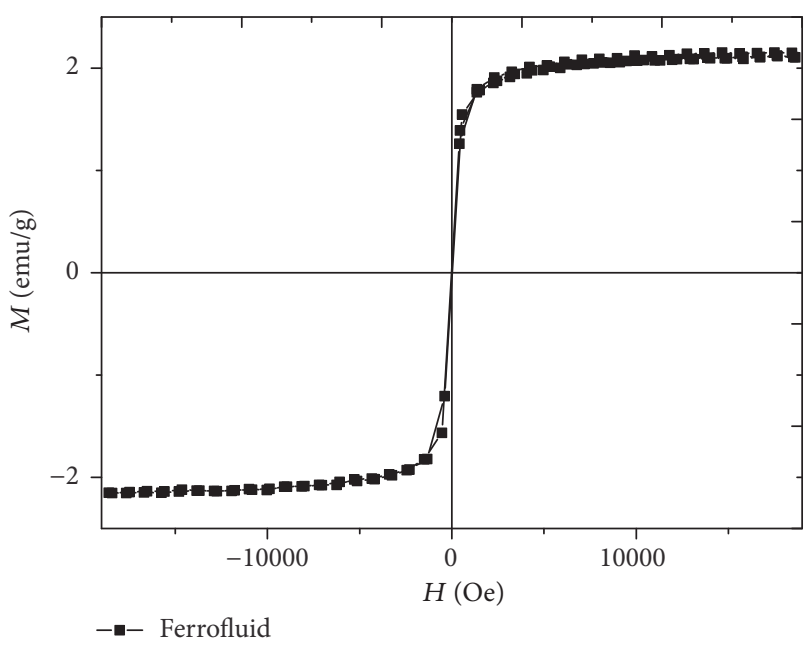

(a)

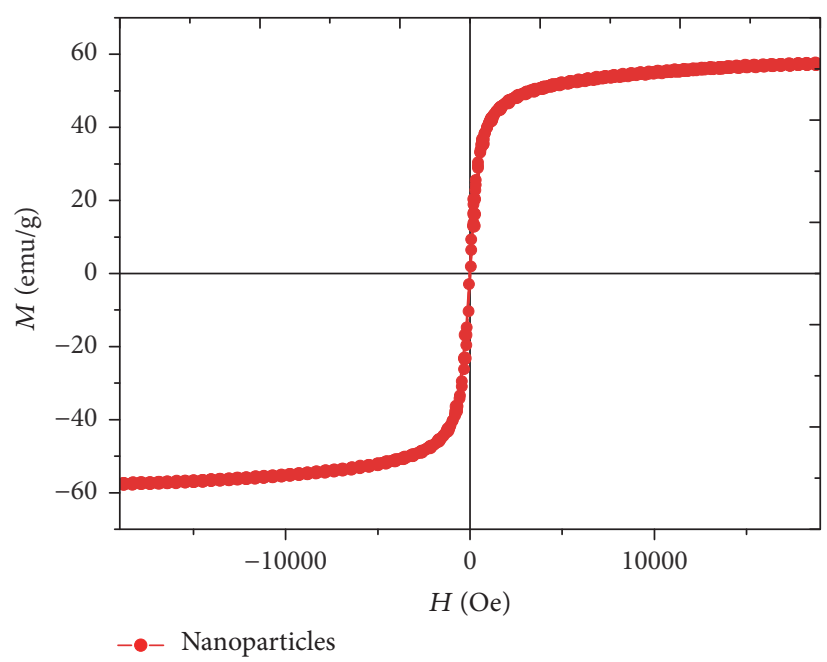

(b)

FIGURE 5: Magnetic hysteresis curve of (a) water-based LTE MNPs ferrofluid and (b) magnetic nanoparticles.

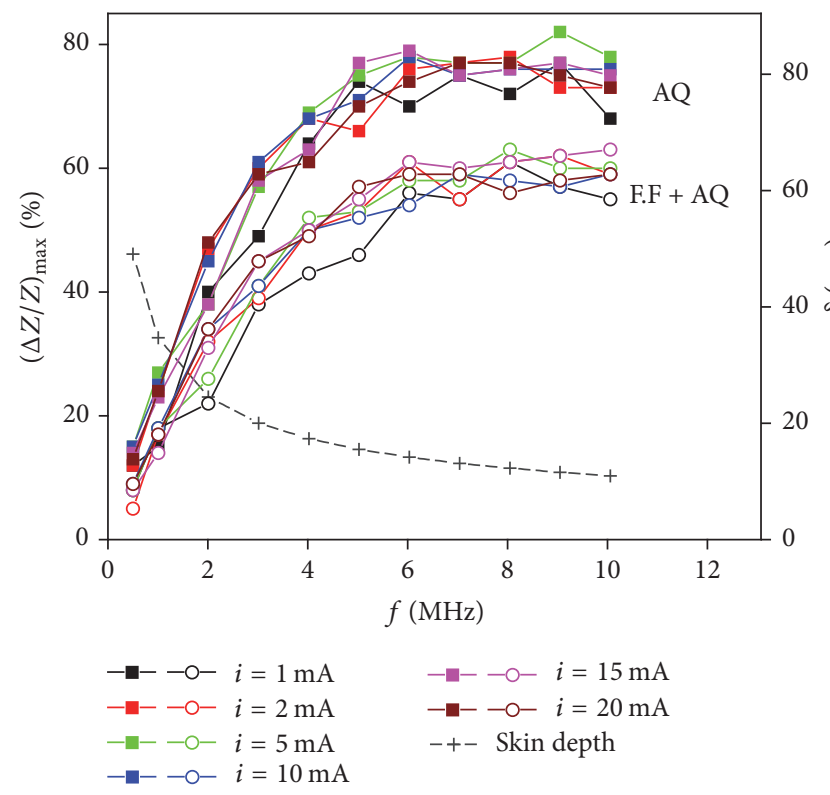

(a)

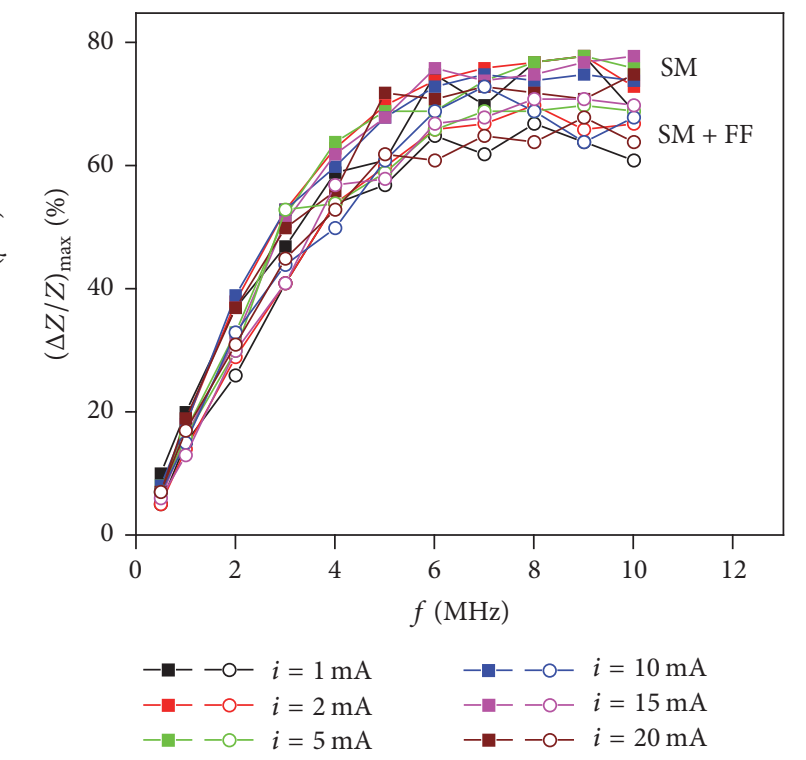

(b)

FIGURE 6: GMI-frequency dependence and frequency dependence of the skin depth in presence and in absence of ferrofluid for (a) asquenched (AQ) and (b) surface modified (SM) $\mathrm{Co}_{68.6} \mathrm{Fe}_{3.9} \mathrm{Mo}_{3.0} \mathrm{Si}_{12.0} \mathrm{~B}_{12.5}$ ribbons.

The concentration range for the detection, optimum current intensity, and magnetic field for maximum sensitivity are still points to investigate for each kind of the biological samples but this direction may result in the development of simple and cheap magnetic biosensor prototype with disposal amorphous ribbon-based GMI sensitive element. As the next step one can plan to create smaller equidistant defects using different parameters of ultrasound treatments for selfassembled defects of desired geometry. In this case one must look at synergetic combinations of the nanoparticles and particular shape/size of the defect in order to insure maximum magnetic flux closing and maximum difference in the GMI response of the sensitive element in the absence and the presence of the magnetic labels creating the stray fields.

\section{Conclusions}

$\mathrm{Co}_{68.6} \mathrm{Fe}_{3.9} \mathrm{Mo}_{3.0} \mathrm{Si}_{12.0} \mathrm{~B}_{12.5}$ ribbons were prepared by rapid quenching technique. Their magnetic and magnetoimpedance properties were studied in as-prepared state and with artificial defects created in the ribbon surface by the ultrasound treatment in the acid. Ultrasound treatment results in appearance of round indentations with average diameter of about 150 micrometers and few $\mu \mathrm{m}$ in depth. 


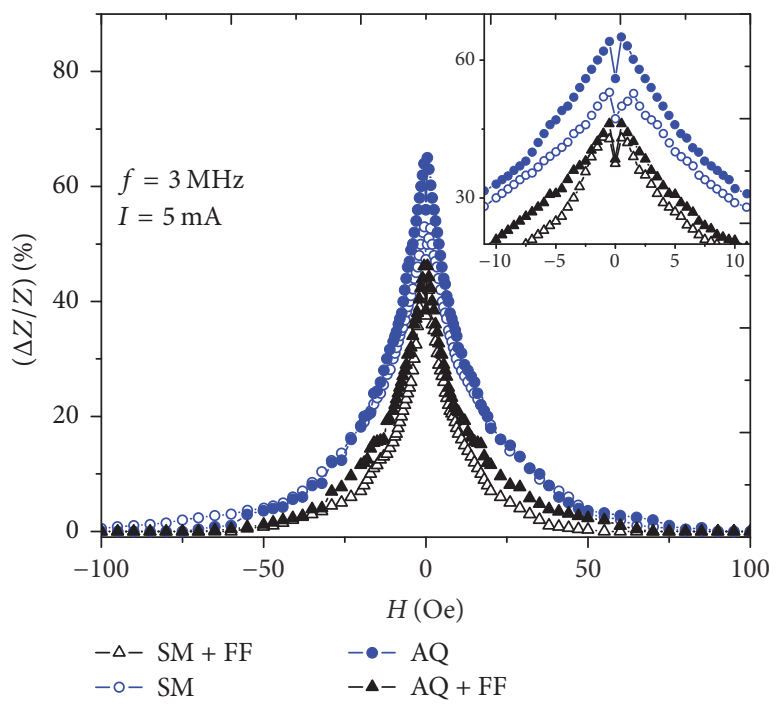

(a)

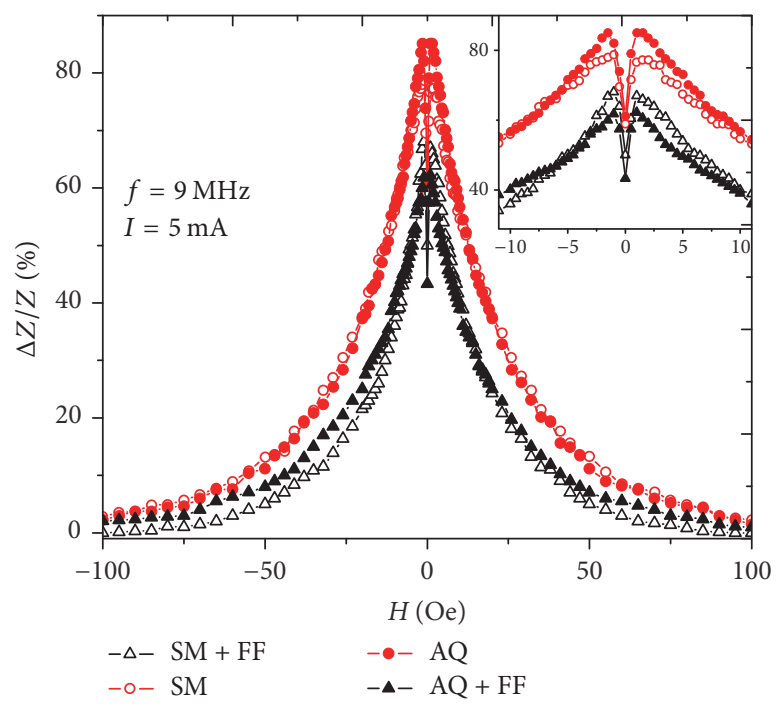

(b)

FIGURE 7: GMI profile of as-quenched (AQ) and surface modified (SM) $\mathrm{Co}_{68.6} \mathrm{Fe}_{3.9} \mathrm{Mo}_{3.0} \mathrm{Si}_{12.0} \mathrm{~B}_{12.5}$ ribbons in presence and in absence of ferrofluid for (a) $f=3 \mathrm{MHz}$ and (b) $f=9 \mathrm{MHz}$. Insets: small field GMI peak resolution.

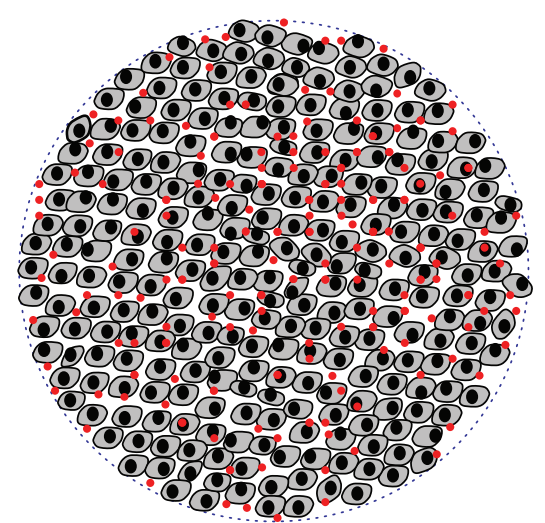

(a)

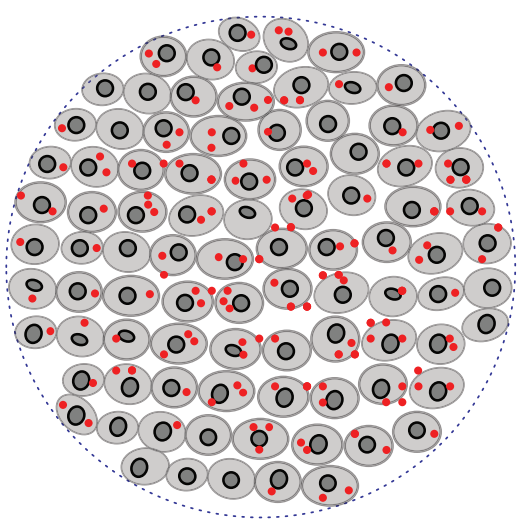

(b)

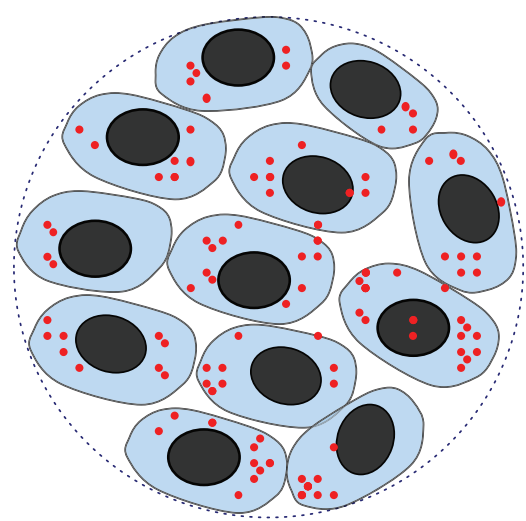

(c)

Figure 8: Schematic description of the comparison of number of cells accommodated in artificial round surface defect with diameter of $150 \mu \mathrm{m}$ : (a) Exophiala nigrum (black yeast), (b) mesenchymal stem cells, and (c) human embryonic kidney cells.

GMI responses were compared for as-quenched and surface modified state in the absence and in presence of LTE MNPs based ferrofluid.

In a whole frequency range $0.5-10 \mathrm{MHz}$ GMI-frequency responses of the as-quenched and surface modified ribbons had very similar shapes but GMI ratios were slightly higher for as-quenched ribbons and all driving currents.

GMI curves appeared to be very much affected by the presence of ferrofluid in AQ and much less in SM ribbon cases. Covering by the ferrofluid resulted in a decrease of GMI ratio in the entire frequency range. The change in the GMI ratio due to the presence of the MNPs can be explained by the effect of their fringe fields. On-purpose creation of round indentations with average diameter of about 150 micrometers makes ribbon less sensitive to the presence of ferrofluid but allows discussing the origin of additional sensitivity to the presence of ferrofluid. Surface modification changes the demagnetizing fields because defects corresponding to rapid solidification are removed and new defects in the shape of indentations are formed. We can suppose that effective flux closing by the magnetic chains of FF is more effective for the as-quenched ribbon. As the next step one can plan to create smaller equidistant defects using different parameters of ultrasound treatments.

For the frequency of $f=3 \mathrm{MHz}$ maximum GMI in presence of FF decreases from $65 \%$ to $46 \%$ and for surface modified ribbon decreases from $53 \%$ to $43 \%$; that is, GMI response of AQ ribbon shows better noise-to-signal characteristics. Also for frequency $f=9 \mathrm{MHz}$ similar conclusion can be made. Obtained results can be useful for the development of simple and cheap magnetic biosensor prototype with disposal amorphous ribbon-based GMI sensitive element for evaluation of the concentration of MNPs in large biocomponents. 


\section{Conflicts of Interest}

The authors declare that there are no conflicts of interest regarding the publication of this paper.

\section{Acknowledgments}

This work was financially supported by the ELKARTEK Grant KK-2016/00030 of the Basque Country Government and the state task of the Ministry of Education and Science of Russia 3.6121.2017/8.9 grants. Some measurements were made at SGIKER UPV-EHU and URFU services. The authors thank A. Larrañaga, O. M. Samatov, and A. I. Medvedev for special support.

\section{References}

[1] R. S. Beach and A. E. Berkowitz, "Giant magnetic field dependent impedance of amorphous FeCoSiB wire," Applied Physics Letters, vol. 64, no. 26, pp. 3652-3654, 1994.

[2] V. E. Makhotkin, B. P. Shurukhin, V. A. Lopatin, P. Y. Marchukov, and Y. K. Levin, "Magnetic field sensors based on amorphous ribbons," Sensors and Actuators A: Physical, vol. 27, no. 1-3, pp. 759-762, 1991.

[3] G. V. Kurlyandskaya, E. Fernández, A. P. Safronov et al., "Giant magnetoimpedance biosensor for ferrogel detection: Model system to evaluate properties of natural tissue," Applied Physics Letters, vol. 106, no. 19, Article ID 193702, 2015.

[4] T. Wang, Y. Zhou, C. Lei, J. Luo, S. Xie, and H. Pu, "Magnetic impedance biosensor: A review," Biosensors and Bioelectronics, vol. 90, pp. 418-435, 2017.

[5] G. V. Kurlyandskaya, V. Fal Miyar, A. Saad, E. Asua, and J. Rodriguez, "Giant magnetoimpedance: A label-free option for surface effect monitoring," Journal of Applied Physics, vol. 101, no. 5, Article ID 054505, 2007.

[6] S. K. Manna and V. Srinivas, "Role of artificially created defects on magnetoimpedance of Co73Fe4.5Mn0.5Nb1.0Si4.2B16.8 ribbon," Journal of Magnetism and Magnetic Materials, 2015.

[7] M. Knobel, L. Kraus, and M. Vazquez, Handbook of Magnetic Materials, edited by K. H. J. Buschow, 2003.

[8] X. Sun, J. Du, Z. Zhu, J. Wang, and Q. Liu, "Enhanced GMI effect in NiZn-ferrite-modified Fe-based amorphous ribbons," Applied Physics A: Materials Science \& Processing, vol. 119, no. 4, pp. 1277-1281, 2015.

[9] E. V. Dirote, New Developments in Nanotechnology Research, Nova Publishers, 2007.

[10] K. Peng, Y. Tang, L. Zhou, J. Tang, F. Xu, and Y. Du, "Influence of corrosion on the magnetic properties of amorphous and nanocrystalline FeZrNbBCu alloy," Physica B: Condensed Matter, vol. 366, no. 1-4, pp. 110-115, 2005.

[11] A. Lovas, É. Kisdi-Koszó, G. Konczos, L. Potocký, and G. Vértesy, "Casting of ferromagnetic amorphous ribbons for electronic and electrotechnical applications," Philosophical Magazine, vol. 61, no. 4, pp. 549-565, 1990.

[12] Z. Lotfollahi, A. García-Arribas, A. Amirabadizadeh, I. Orue, and G. V. Kurlyandskaya, "Comparative study of magnetic and magnetoimpedance properties of CoFeSiB-based amorphous ribbons of the same geometry with Mo or W additions," Journal of Alloys and Compounds, vol. 693, pp. 767-776, 2017.

[13] E. Müller, C. Oestreich, U. Popp, G. Michel, G. Staupendahl, and K.-H. Henneberg, "Characterization of Nanocrystalline Oxide
Powders Prepared by CO2 Laser Evaporation," KONA Powder and Particle Journal, vol. 13, pp. 79-90, 1995.

[14] H.-D. Kurland, J. Grabow, G. Staupendahl, W. Andrä, S. Dutz, and M. E. Bellemann, "Magnetic iron oxide nanopowders produced by $\mathrm{CO} 2$ laser evaporation," Journal of Magnetism and Magnetic Materials, vol. 311, no. 1, pp. 73-77, 2007.

[15] V. V. Osipov, V. V. Platonov, M. A. Uimin, and A. V. Podkin, "Laser synthesis of magnetic iron oxide nanopowders," Technical Physics, vol. 57, no. 4, pp. 543-549, 2012.

[16] A. P. Safronov, I. V. Beketov, S. V. Komogortsev et al., "Spherical magnetic nanoparticles fabricated by laser target evaporation," AIP Advances, vol. 3, no. 5, Article ID 052135, 2013.

[17] A. Amirabadizadeh, Z. Lotfollahi, and A. Zelati, "Giant magnetoimpedance effect of Co68.15 Fe4.35 Si12.5 B15 amorphous wire in the presence of magnetite ferrofluid," Journal of Magnetism and Magnetic Materials, vol. 415, pp. 102-105, 2016.

[18] W. B. Pearson, A Handbook of Lattice Spacings and Structures of Metals and Alloys: International Series of Monographs on Metal Physics and Physical Metallurgy, vol. 4, Elsevier, 2013.

[19] A. A. Chlenova, E. A. Stepanova, E. V. Golubeva, and Z. Lotfollahi, "Magnetoimpedance and magnetic properties of Co72Fe5Ni10Cr5Si7B3 amorphous ribbons in different states," Journal of Magnetism and Magnetic Materials, vol. 440, pp. 210212, 2017.

[20] U. Köster, "Surface crystallization of metallic glasses," Materials Science and Engineering: A Structural Materials: Properties, Microstructure and Processing, vol. 97, no. C, pp. 233-239, 1988.

[21] K. Russew and L. Stojanova, "Properties and applications of amorphous metallic alloys," in Glassy Metals, pp. 217-241, Springer, Berlin, Germany, 2016.

[22] J. Li, X. M. Gong, Y. Q. Lin et al., "Investigation into loss in ferrofluid magnetization," AIP Advances, vol. 4, no. 7, Article ID 077123, 2014

[23] G. V. Kurlyandskaya, M. L. Sánchez, B. Hernando, V. M. Prida, P. Gorria, and M. Tejedor, "Giant-magnetoimpedance-based sensitive element as a model for biosensors," Applied Physics Letters, vol. 82, no. 18, pp. 3053-3055, 2003.

[24] A. Hubert and R. Schäfer, Magnetic Domains: The Analysis of Magnetic Microstructures, Springer Science \& Business Media, 2008 .

[25] J. Devkota, N. T. Huong, H. Srikanth, and M.-H. Phan, "Magnetoimpedance-based probe of various concentrations of corrosive chemicals," IEEE Transactions on Magnetics, vol. 50, no. 6, 2014.

[26] J. P. Novoselova, A. P. Safronov, O. M. Samatov et al., "Laser target evaporation $\mathrm{Fe} 2 \mathrm{O} 3$ nanoparticles for water-based ferrofluids for biomedical applications," IEEE Transactions on Magnetics, vol. 50, no. 11, 2014.

[27] J. J. Minguell, A. Erices, and P. Conget, "Mesenchymal stem cells," Experimental Biology and Medicine, vol. 226, no. 6, pp. 507-520, 2001

[28] G. V. Kurlyandskaya, I. P. Novoselova, V. V. Schupletsova et al., "Nanoparticles for magnetic biosensing systems," Journal of Magnetism and Magnetic Materials, vol. 431, pp. 249-254, 2017. 


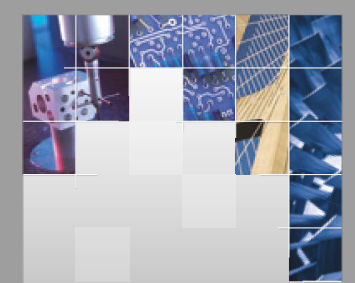

\section{Enfincering}
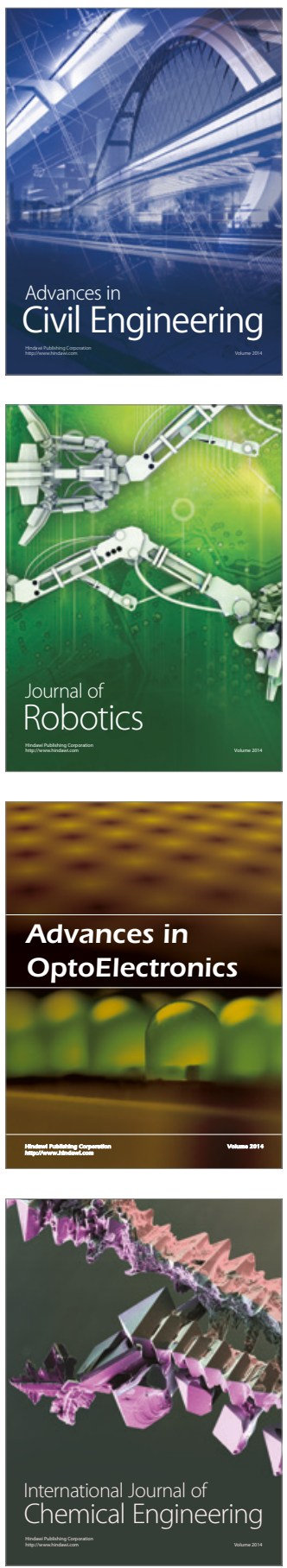

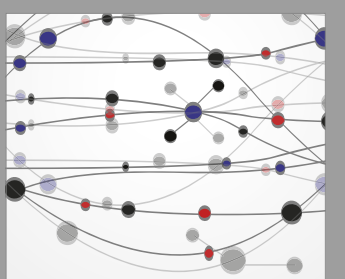

The Scientific World Journal

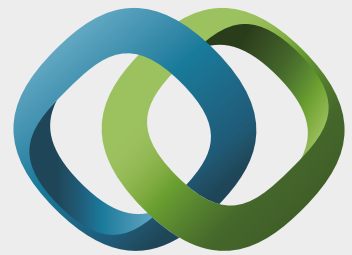

\section{Hindawi}

Submit your manuscripts at

https://www.hindawi.com
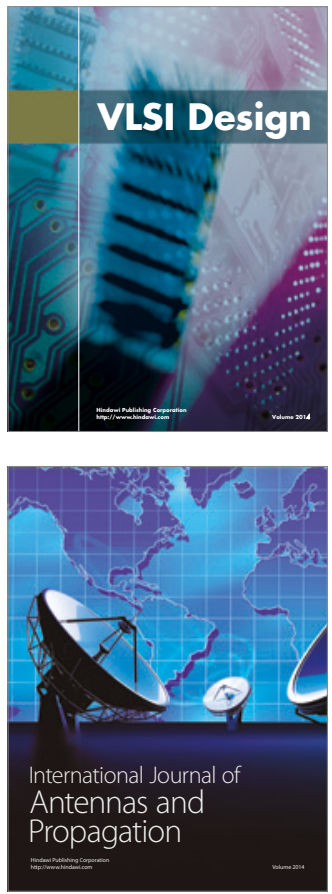

\section{Rotating}

Machinery
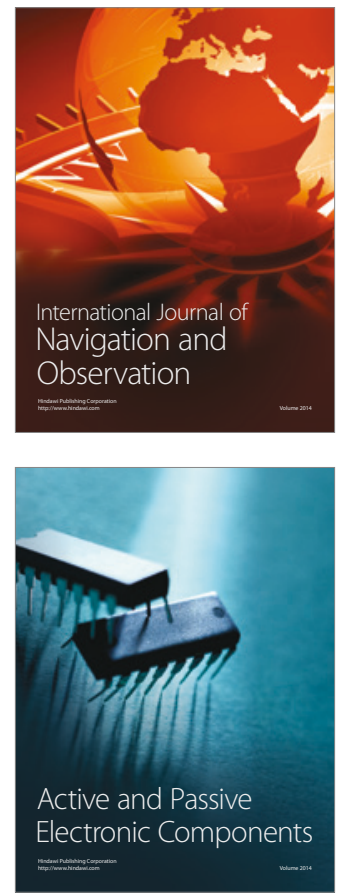
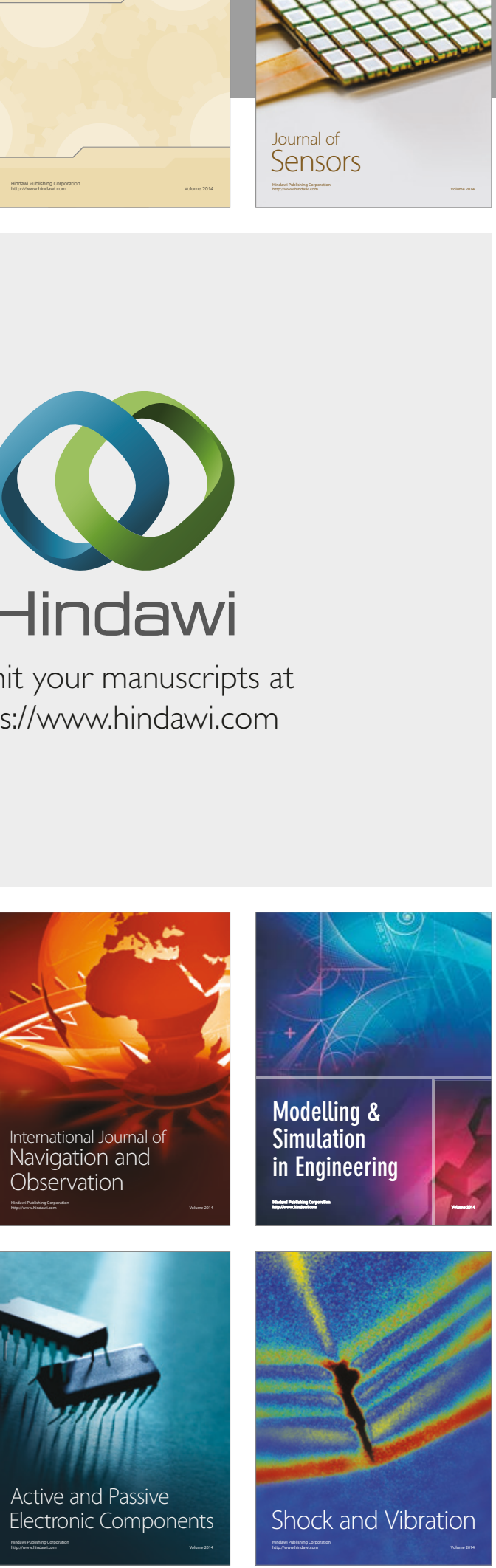
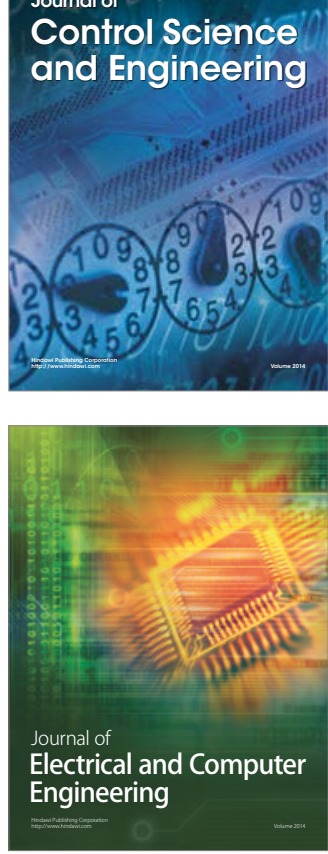

Distributed

Journal of

Control Science

and Engineering
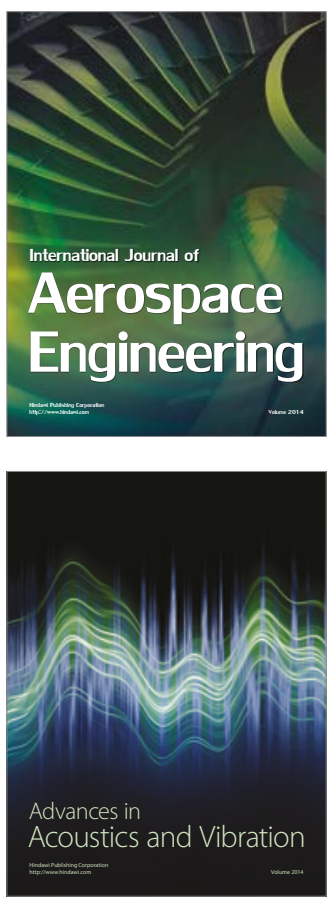

Sensor Networks 